\title{
Effect of Tiling in Row Mean of Column Transformed Image as Feature Vector for Iris Recognition with Cosine, Hadamard, Fourier and Sine Transforms
}

\author{
H. B. Kekre \\ Senior Professor \\ MPSTME, SVKM's NMIMS \\ Deemed to be University \\ Mumbai
}

\author{
Sudeep D. Thepade \\ Professor, Computer \\ Engineering Dept., \\ PCCOE, Pune.
}

\author{
Donovan Pereira, Kiran \\ Rohra \\ Student, BTech Computer \\ Engineering, \\ MPSTME, SVKM's NMIMS \\ Deemed to be University \\ Mumbai
}

\begin{abstract}
Iris recognition is a biometric authentication method that uses pattern-recognition techniques based on high-resolution images of the irises of an individual's eyes. Iris recognition has been a fast growing, challenging and interesting area in real-time applications. A large number of iris recognition algorithms have been developed for decades. This paper presents the techniques of iris recognition using image transforms such as Cosine transform, Sine transform, Fourier transform and Hadamard transform. Here iris recognition is done using the image feature vector set extracted as row mean of transformed column iris image. Image tiling is further used for feature extraction for each transform and the performance is compared with the single tile based iris recognition method. Parameters such as False Acceptance Rate and Genuine Acceptance Rate are used to test the performance of the techniques. The results have shown that the proposed Iris recognition methods performs better with increased number of tiles of Iris image up to certain extent of tiling.
\end{abstract}

\section{Keywords}

Iris Recognition, Row Mean, Image Tiling, Image Transforms.

\section{INTRODUCTION}

The iris is a thin, circular structure in the eye, responsible for controlling the diameter and size of the pupils and thus the amount of light reaching the retina [1]. A primary visible characteristic is the trabecular meshwork, a tissue that gives the appearance of dividing the iris in radial fashion [2]. Other visible characteristics include rings, furrows, freckles, and the corona. Iris' are composed before birth and, remain unchanged throughout an individual's lifetime; until accidently the eyeball is injured. Iris patterns are very complex, which carry an astonishing amount of information and it has over 200 unique spots [2]. The fact that an individual's right and left eye differ and that patterns are easy to capture, make iris-scan technology very resistant to false matching and fraud. Iris Recognition has a number of applications in every authenticating system such as computer logins, national border surveillance, forensics, personal certificates etc $[3,4]$.

\section{ROW MEAN OF TRANSFORMED COLUMN IMAGE}

In the procedure of computing the row mean of a transformed column iris image, selected transform is applied to every column of the iris image $[5,6]$. Then the mean of the values in each row of the transformed column matrix is computed. These row means now form a column vector $(\mathrm{n} \times 1$ where $\mathrm{n}$ is the number of rows in the transform matrix). This column vector is the feature vector for the iris image sample.

The feature vectors for all the images are calculated and used for comparison with the feature vector of the query image instead of comparing actual pixel intensity values of the images [7]. Since the size of the feature vector ( $\mathrm{n} \times 1)$ is less than the size of the actual image ( $\mathrm{x} n$ ), the computations are reduced and the code runs at a faster rate than normal pixel by pixel comparison [8].

Here four different image transforms alias Cosine transform $[6,9,10]$, Sine transform [9], Hadamard transform [11, 12, 6] and Fourier transform [3] are used. The process of computing row mean of transformed column iris image is as shown in figure 1.

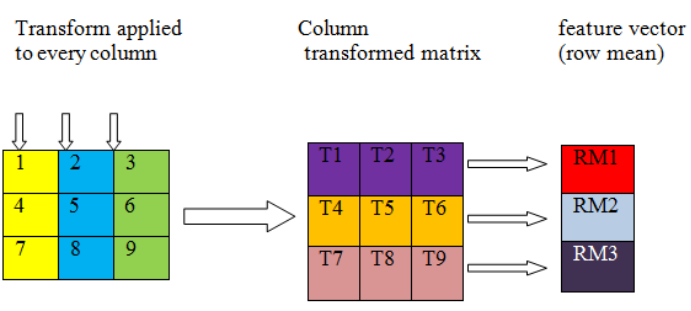

Figure 1 - Row Mean of Column Transformed Iris Images

\section{IRIS DATABASE}

The techniques proposed are tested on an Iris Database created at Palacky University, Moravia, Czech Republic. This database has $6 \times 64$ (i.e. 3x64 left iris and 3x64 right iris) images (each of size 576 pixels by 768 pixels), corresponding to 64 persons, including both males and females [4, 13]. Sample images are shown in figure 2 and figure 3. The irises were scanned by TOPCON TRC50IA optical device connected with SONY DXC- 950P 3CCD camera [13]. 
Peron 1:

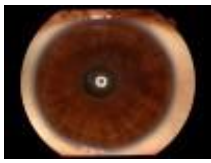

1.png

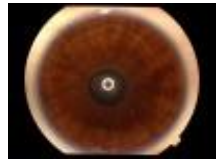

2.png

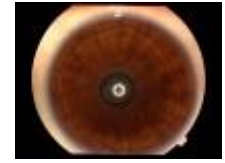

3.png
Figure 2 - Sample set of Left Iris Images of Person 1

Person 1:

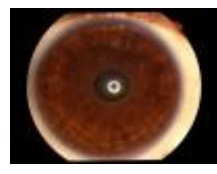

1.png

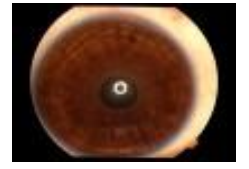

2.png

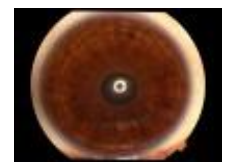

3.png
Figure 3 - Sample set of Right Iris Images of Person 1

\section{IRIS RECOGNITION USING ROW MEAN OF TRANSFORMED COLUMNS}

In iris recognition based on one tiled image (1T) the entire image as a whole is considered, as shown in figure 4 . The transforms DCT [6, 9,10], DST [9], Hadamard [11, 12, 6] and FFT [3] are applied on the columns of the image, one column at a time. Then, row mean of the column transformed images is calculated. Now the $256 \times 256 \times 3$ sized image is converted into its feature vector (FT) of size $256 \times 1 \times 3$. In this way, feature vector is calculated for all the images in the database and absolute difference between the FV of the query image and that of the database images is calculated.

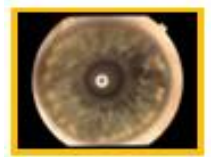

Figure 4 - Image tiling in to 1 part

In iris recognition based on four tiled image (4T), as shown in Figure 5, the image is divided in to 4 parts each of size $128 \times 128$. Now, row mean of transformed column images are calculated (separately for each part). Thus, it results in 4 feature vectors for each plane of the image, each of size $128 \times 1$. These $4 \mathrm{FVs}$ are then combined together [5] to give a resultant FV of size $128 \times 4 \times 3$.

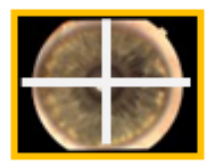

Figure 5 - Image tiling in to 4 parts

In iris recognition based on sixteen tiled image (16T), as depicted in Figure 6, the image is divided in to 16 parts each of size $64 \times 64$. The, row mean of transformed column images are calculated (separately for each part). Thus, it results in 16 feature vectors for each plane of the image, each of size $64 \times 1$. These $16 \mathrm{FVs}$ are then combined together [5] to give a resultant FV of size $64 \times 16 \times 3$.

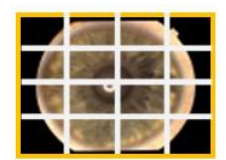

Figure 6 - Image tiling in to 16 parts

\section{RESULTS AND OBSERVATIONS}

Observation of all techniques used on the entire image without tiling (GAR Values are used for Comparisons)

Table 1 - Observations of the Various Techniques used (on the whole image without tiling; GAR values are depicted in the table)

From Table 1, it can be inferred that using the full image as the feature vector shows the best results. However, this technique has the major drawback of being extremely time

\begin{tabular}{|c|c|c|}
\hline Techniques & $\begin{array}{c}\text { Left Iris } \\
\text { Database }\end{array}$ & $\begin{array}{c}\text { Right Iris } \\
\text { Database }\end{array}$ \\
\hline $\begin{array}{c}\text { Full Image as Feature } \\
\text { Vector }\end{array}$ & 0.7361 & 0.7535 \\
\hline $\begin{array}{c}\text { Row Mean of Column - } \\
\text { DCT }\end{array}$ & 0.6285 & 0.691 \\
\hline $\begin{array}{c}\text { Row Mean of Column - } \\
\text { DST }\end{array}$ & 0.6146 & 0.6667 \\
\hline $\begin{array}{c}\text { Row Mean of Column - } \\
\text { Hadamard }\end{array}$ & 0.6076 & 0.6771 \\
\hline $\begin{array}{c}\text { Row Mean of Column - } \\
\text { FFT }\end{array}$ & 0.6111 & 0.7396 \\
\hline
\end{tabular}

consuming. To overcome this disadvantage, the paper proposes a solution to minimize the size of the feature vector. Also as it can be seen in the figure 7, it can be concluded that the best result is obtained for the images in the left iris database using DCT transform while that for images in the right iris database, is obtained by using the FFT transform.

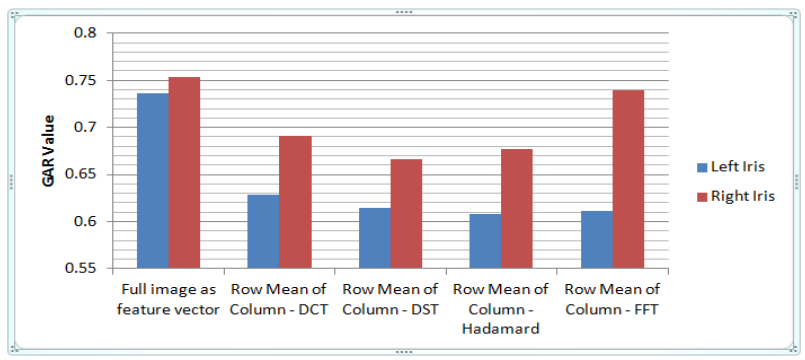

Figure 7 - Techniques used for computing GAR values for image as a whole

Observations of DCT computations with Image Tiling 
Table 2 - Observations of DCT Row Mean of Column Computations with Image Tiling (GAR values are depicted in the table)

\begin{tabular}{|c|c|c|}
\hline Techniques & $\begin{array}{c}\text { Left Iris } \\
\text { Database }\end{array}$ & $\begin{array}{c}\text { Right Iris } \\
\text { Database }\end{array}$ \\
\hline Entire Image & 0.6285 & 0.691 \\
\hline 4 Tiled Image & 0.6597 & 0.7066 \\
\hline $\begin{array}{c}\text { 16 Tiled } \\
\text { Image }\end{array}$ & 0.6771 & 0.7135 \\
\hline
\end{tabular}

From Table 2, it can be inferred that the accuracy of the result increases with image tiling from 1 part to 4 parts and then to 16 parts. Hence the best result is shown when the image is tiled in to 16 parts. Also, results obtained are better for images in the right iris database. The same can be seen in Figure 8, in the form of a bar chart.

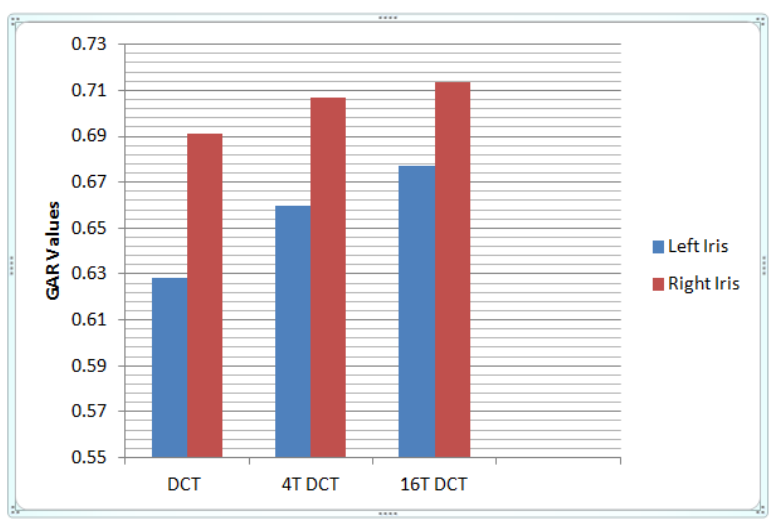

Figure 8 - Iris Recognition using DCT transform with image tiling

Observations of DST Computations with Image Tiling

\section{Table 3 - Observations of DST Row Mean of Column Computations with Image Tiling (GAR values are depicted in the table)}

By observing table 3 , it can be seen that the accuracy of the

\begin{tabular}{|c|c|c|}
\hline Techniques & $\begin{array}{c}\text { Left Iris } \\
\text { Database }\end{array}$ & $\begin{array}{l}\text { Right Iris } \\
\text { Database }\end{array}$ \\
\hline Entire Image & 0.6146 & 0.6667 \\
\hline 4 Tiled Image & 0.6632 & 0.7031 \\
\hline $\begin{array}{c}\text { 16 Tiled } \\
\text { Image }\end{array}$ & 0.6962 & 0.7188 \\
\hline
\end{tabular}

result increases with image tiling from 1 part to 4 parts and then to 16 parts wherein the accuracy goes up to $70 \%$. Hence the best result is shown when the image is tiled in to 16 parts.
Also, results obtained are better for images in the right iris database.

The same can be referred from Figure 9.

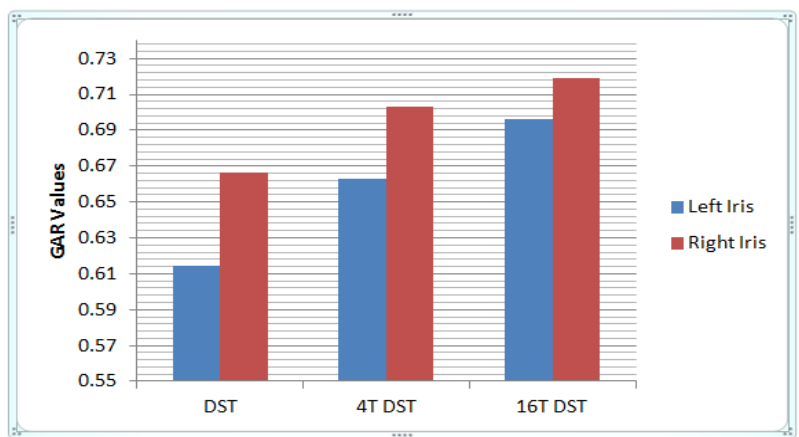

Figure 9 - Iris recognition using DST Transform with Image Tiling

Observations of Hadamard Computations with Image Tiling

Table 4 - Observations of Hadamard Row Mean of Column Computations with Image Tiling (GAR values are depicted in the table)

\begin{tabular}{|c|c|c|}
\hline Technique & Left Iris Database & Right Iris Database \\
\hline Entire Image & 0.6076 & 0.6771 \\
\hline $\begin{array}{c}\text { 4 Tiled } \\
\text { Image }\end{array}$ & 0.6615 & 0.7014 \\
\hline $\begin{array}{c}\text { 16 Tiled } \\
\text { Image }\end{array}$ & 0.6684 & 0.7066 \\
\hline
\end{tabular}

Looking at the Table 4, it can be observed that the accuracy of the result increases with image tiling from 1 part to 4 parts and then to 16 parts. Hence the best result is shown when the image is tiled in to 16 parts. Also, results obtained are better for images in the right iris database wherein the accuracy reaches up to $70.66 \%$. The same can also be seen in Figure 10

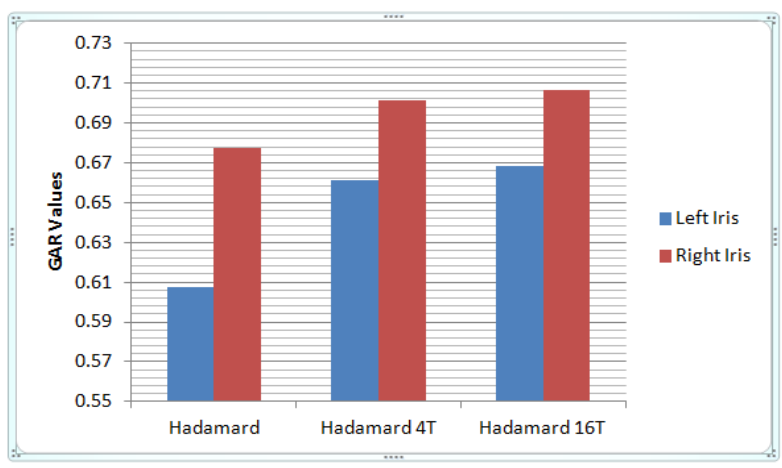

Figure 10 - Iris recognition using Hadamard transform with image tiling 
Observation of FFT Computations with Image Tiling

Table 5 - Observations of FFT Row Mean of Column Computations with Image Tiling (GAR values are depicted in the table)

\begin{tabular}{|l|l|l|}
\hline Techniques & $\begin{array}{l}\text { Left Iris } \\
\text { Database }\end{array}$ & $\begin{array}{l}\text { Right Iris } \\
\text { Database }\end{array}$ \\
\hline Entire Image & 0.6111 & 0.7344 \\
\hline 4 Tiled Image & 0.6337 & 0.6892 \\
\hline 16 Tiled Image & 0.7049 & 0.7222 \\
\hline
\end{tabular}

On observing Table 5, it can be concluded that even though the accuracy of the result increases with image tiling from 4 parts to 16 parts, the best result is obtained when the entire image is considered as a single tile for the images from the right iris database. However, for images from the left iris database, the accuracy of the result increases with image tiling from 1 part to 4 parts and then to 16 parts. Here too, results obtained are better for images in the right iris database.

The same can be concluded from Figure 11 .

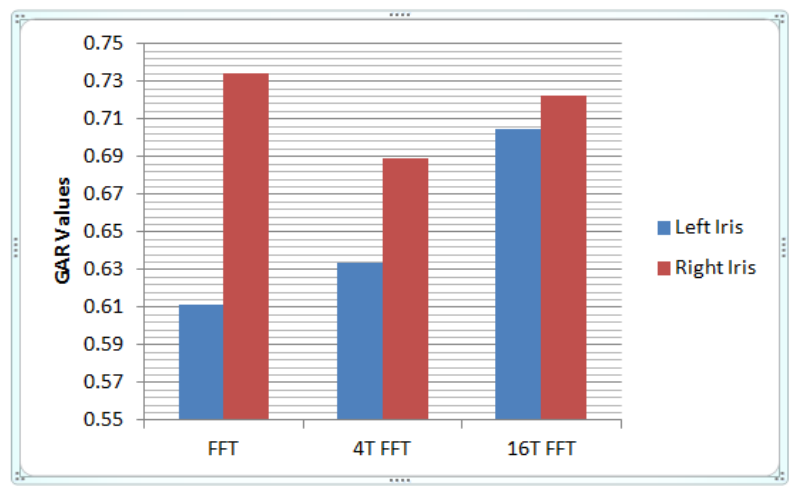

Figure 11 - Iris recognition using FFT transform with image tiling

\section{CONCLUSION}

From the observations, it can be concluded that Pixel by pixel gives the best result compared to row mean of column transformed images, but the computation complexity increases, i.e. for pixel by pixel comparison, assuming a 256x256 image size, the number of comparisons made are $256 \times 256 \times 3$ (considering the 3 planes of the image), whereas, if we use the feature vector computed by row mean of a column transformed image, then the total number of comparisons required are $256 \times 1 \times 3$. Hence, the computations have been reduced by a factor of 256 .

Also, when the full image is considered as a feature vector for comparison, a little lateral shift or movement in the image can produce erroneous results, but this is overcome by row mean of column transformed images, which works by considering columns.
For column-transformed images, DCT gives the best result on left iris images while FFT gives best for right iris images.

Tiling of the image increases the performance of the proposed iris recognition technique. Increasing the amount of tiling to only a certain extent can still increase performance. This implies that considering a sixteen tiled image gives better results than considering a four tiled image which in turn gives a better result than no tiling. The above tiling concept is contradicted by FFT for right iris images.

\section{REFERENCES}

[1] http://en.wikipedia.org/wiki/Iris_recognition(referred as on 23 December 2011).

[2] Mihran Tuceryan, Anil K Jain, "Chapter 2.1, Texture Analysis", The Handbook of Pattern Recognition and Computer Vision (2nd Edition), by C. H. Chen, L. F. Pau, P. S. P. Wang (eds.), pp. 207-248, World Scientific Publishing Co., 1998.

[3] R.C. Gonzalez, R.E. Woods "Digital Image Processing", Third Edition, 2008, Upper Saddle River, New Jersey 07458, Pearson Publication.

[4] Dr. H B Kekre, Dr. Sudeep Thepade, Juhi Jain, Naman Agrawal, "IRIS Recognition using Texture Features Extracted from Haarlet Pyramid", International Journal of Computer Applications (0975-8887), Volume 11No.12, December 2010.

[5] Dr. H.B.Kekre, Sudeep D. Thepade, Varun K. Banura, "Augmentation of Colour Averaging Based Image Retrieval Techniques using Even part of Images and Amalgamation of feature vectors", International Journal of Engineering Science and Technology (IJEST), Volume 2, Issue 10, (ISSN: 0975-5462).

[6] Dr. H.B.Kekre, Sudeep D. Thepade, Akshay Maloo "Performance Comparison for Face Recognition using PCA, DCT \& Walsh Transform of Row Mean and Column Mean", ICGST International Journal on Graphics, Vision and Image Processing (GVIP), Volume 10, Issue II, Jun.2010, pp.9-18.

[7] Hirata K. and Kato T. "Query by visual example content-based image retrieval", In Proc. of Third International Conference on Extending Database Technology, EDBT'92, 1992, pp 56-71.

[8] H B Kekre, Sudeep D. Thepade, Archana A. Athawale, Paulami Shah, "Image Retrieval using Fractional Energy of Row Mean of Column Transformed Image with Six Orthogonal Image Transforms", International Journal of Soft Computing and Engineering (IJSCE), ISSN: 22312307, Volume-1, Issue-4, September 2011.

[9] Dr. H.B.Kekre, Sudeep D. Thepade, "Improving the Performance of Image Retrieval using Partial Coefficients of Transformed Image", International Journal of Information Retrieval, Serials Publications, Volume 2, Issue 1, 2009, pp. 72-79.

[10] Dr. H.B.Kekre, Sudeep D. Thepade, Akshay Maloo "Performance Comparison of Image Retrieval Using Fractional Coefficients of Transformed Image Using DCT, Walsh, Haar and Kekre's Transform", CSC International Journal of Image Processing (IJIP), Volume 4, Issue 2, pp 142-157, Computer Science Journals. 
[11] Dr. H B Kekre, Sudeep D. Thepade, Varun K. Banura, "Image Retrieval using Texture Patterns generated from Walsh-Hadamard Transform Matrix and Image Bitmaps", Springer International Conference on Technology Systems and Management (ICTSM 2011), MPSTME and DJSCOE, Mumbai, 25-27 Feb 2011.

[12] Dr. H B Kekre, Sudeep D. Thepade, Varun K. Banura, "Image Retrieval using Shape Texture Patterns generated from Walsh-Hadamard Transform and Gradient Image Bitmaps", International Journal of Computer Science and Information Security (IJCSIS), Volume 8, Number 9, 2010.pp.76-82.

[13] http://www.advancedsourcecode.com/irisdatabase.asp for Palacky University iris database.

\section{AUTHOR BIOGRAPHIES}

Dr. H. B. Kekre has received B.E. (Hons.) in Telecomm Engineering from Jabalpur University in 1958, M. Tech (Industrial Electronics) from IIT Bombay in 1960, M.S.Engg. (Electrical Engg.) from University of Ottawa in 1965 and Ph.D. (System Identification) from IIT Bombay in $1970 \mathrm{He}$ has worked as Faculty of Electrical Engg. and then HOD Computer Science and Engg. at IIT Bombay. For 13 years, he was working as a professor and head in the Department of Computer Engg. at Thadomal Shahani Engineering College, Mumbai. Now, he is Senior Professor at MPSTME, SVKM"es NMIMS. He has guided 17 Ph.Ds, more than 100 M.E./M.Tech and several B.E./ B.Tech projects. His areas of interest are Digital Signal processing, Image Processing and Computer Networking. He has more than 300 papers in National / International Conferences and Journals to his credit. He was Senior Member of IEEE. Presently He is Fellow of IETE and Life Member of ISTE Recently seven students working under his guidance have received best paper awards. Currently 10 research scholars are pursuing Ph.D. program under his guidance.

Dr. Sudeep D. Thepade has Received B.E.(Computer) degree from North Maharashtra University with Distinction in 2003. M.E. in Computer Engineering from University of Mumbai in 2008 with Distinction, Ph.D. from SVKM's NMIMS University, Mumbai in 2011. He has more than 08 years of experience in teaching and industry. He was Lecturer in Dept. of Information Technology at Thadomal Shahani Engineering College, Bandra(w), Mumbai for nearly 04 years. $\mathrm{He}$ also worked as as Associate Professor and $\mathrm{HoD}$ in Computer Engineering at SVKM's NMIMS University, Vile Parle(w), Mumbai, INDIA. Currently working as a Professor in Computer Engineering in PCCOE, Pune. He is member of International Association of Engineers (IAENG) and International Association of Computer Science and Information Technology (IACSIT), Singapore.

His areas of interest are Image Processing and Computer Networks. He has more than 135 papers in National/International Conferences/Journals to his credit with a Best Paper Award at International Conference SSPCCIN2008, Second Best Paper Award at ThinkQuest-2009 National Level paper presentation competition for faculty and Best Paper Award at Springer International Conference on Contours of Computing Technology (ICCCT-2010).

Donovan Pereira is currently pursuing B.Tech. (Computer Engineering) from MPSTME, SVKM's NMIMS University, Mumbai. His areas of interest are Image Processing, Marketing, Computer Networks, Information Storage and Management and Biometrics.

Kiran Rohra is currently pursuing B.Tech. (Computer Engineering) from MPSTME, SVKM's NMIMS University, Mumbai. Her areas of interest are Image Processing, Database Management System, E-Commerce, Biometrics and Computer Networks. 